\title{
Effect of Carbohydrate Intake During Recovery From Eccentric Exercise on Interleukin-6 and Muscle-Damage Markers
}

\author{
Mary P. Miles, Sherri D. Pearson, Jan M. Andring, \\ Jessy R. Kidd, and Stella L. Volpe
}

\begin{abstract}
The purpose of this investigation was to determine whether carbohydrate supplementation during the first $2 \mathrm{~d}$ postexercise recovery influenced the inflammation (IL-6, C-reactive protein [CRP], and cortisol) and muscle-damage responses. Eight participants performed a high-force eccentric elbow-flexion exercise to induce muscle soreness and inflammation and then consumed carbohydrate $\left(0.25 \mathrm{~g} \cdot \mathrm{kg}^{-1} \cdot \mathrm{h}^{-1}\right)$ or an equal volume of placebo during hours 0-12 and 24-36 postexercise in a double-blind, crossover protocol. Muscle soreness; midbrachial arm circumference; blood glucose, IL-6, CRP, cortisol, and creatine-kinase (CK) activity; and maximal force production were measured preexercise and $4,8,12$, 24,48 , and $120 \mathrm{~h}$ postexercise. Plasma IL-6 increased, $F(5)=5.27, P<0.05,8$ $\mathrm{h}$ postexercise, with no difference between carbohydrate and placebo conditions. Changes in muscle soreness, arm circumference, strength, and serum CK activity were consistent with small amounts of muscle damage and did not differ between conditions. The authors conclude that carbohydrate supplementation during recovery from soreness-inducing exercise does not influence the delayed IL-6 response temporally linked to inflammation or indications of muscle damage. Thus, increased carbohydrate consumption at levels consistent with recommendations for replenishing glycogen stores does not impair or promote the immune and muscle responses.
\end{abstract}

Key Words: C-reactive protein, cortisol, creatine kinase

Interleukin-6 (IL-6) is a cytokine with many important roles related to exercise. The mechanism inducing production of IL-6 is different when it is produced in response to muscle contraction than when it is produced in response to muscletissue damage and inflammation. After muscle-damaging exercise, initiation of muscle-tissue injury by high-force eccentric contractions promotes infiltration by inflammatory cells $(11,19)$. Those cells, in conjunction with local muscle, endothelial, and satellite cells, produce an array of cytokines to regulate the

Miles, Pearson, Andring, and Kidd are with the Dept. of Health and Human Development, Montana State University, Bozeman MT 59717. Volpe is with the Div. of Biobehavioral and Health Sciences, University of Pennsylvania, Philadelphia, PA 19104-6096. 
inflammatory process, including tumor-necrosis factor- $\alpha$ (TNF- $\alpha$ ), IL-1 $\beta$, and IL-6 $(5,30,32,35)$. The inflammatory process is initiated by "alarm cytokines" TNF- $\alpha$ and IL-1 $\beta$, which induce synthesis of IL- 6 . The role of IL- 6 during the inflammatory process includes providing feedback inhibition to disallow synthesis of the alarm cytokines and to induce synthesis of anti-inflammatory mediators IL-1 receptor antagonist (IL-1ra) and IL-10 (32). In contrast, IL-6 is produced in skeletal muscle in response to contractile activity, an effect that occurs without production of TNF- $\alpha$ (33). In addition, the relative increase in IL-6 is lowest after high-force resistance or discontinuous exercise and several orders of magnitude lower than the increase on completion of prolonged endurance exercise $(13,23,29)$.

Carbohydrate supplementation during endurance exercise modulates the cytokine response, most notably by decreasing the IL-6 response $(23,25,26)$. Consumption of about $1 \mathrm{~g}$ of carbohydrate per kilogram of body mass per hour attenuates the IL- 6 and IL-1ra responses to prolonged endurance exercise (23, $25,26)$. Attenuation of the IL-6 response during prolonged exercise is likely to be because of the glucoregulatory role IL-6 plays rather than its inflammationmediating role. Carbohydrate does not influence synthesis of cytokines associated with the initiation of inflammation upstream of IL-6, such as IL-1 $\beta$ (26). Thus, the influence of carbohydrate on IL-6 during endurance exercise is likely to differ from the influence of carbohydrate on IL-6 after muscle-damaging exercise. Of the cytokines with roles in the inflammatory cascade, carbohydrate during exercise exerts its influence starting with IL- 6 and continuing to its downstream effectors, IL-1 receptor antagonist (IL-1ra) and IL-10 (33).

Glucose ingestion is known to promote inflammation in many instances, an effect that occurs upstream of IL-6 in the inflammatory cascade. Aljada et al. (1) measured increased proinflammatory transcription factor, nuclear factor $\mathrm{kB}$ (NF$\kappa \mathrm{B}$ ), and TNF- $\alpha$ gene expression in mononuclear cells collected from healthy human subjects after ingestion of $75 \mathrm{~g}$ of glucose. Gonzalez et al. (14) found that hyperglycemia enhanced TNF- $\alpha$ synthesis in obese but not normal-weight women. In this case, the stimulating influence of carbohydrate occurred only when inflammation had been activated, as it is with obesity. Furthermore, a high-carbohydrate, energy-balanced diet was found to increase basal levels of C-reactive protein (CRP) (18). Toward the end of the inflammatory cascade, CRP is an acute-phase protein induced by inflammatory cytokines, particularly IL-6. Given this evidence for carbohydrate ingestion to increase inflammatory mediators at both the beginning and end of the inflammatory cascade, it is hypothesized that increasing carbohydrate intake during active inflammation driven by TNF- $\alpha$ will not decrease and might increase IL-6. An increase in tissue-level inflammation could increase the muscledamage response. This is in contrast to the influence of carbohydrate ingestion on IL-6 produced during endurance exercise.

The influence of carbohydrate intake during recovery from exercise on the delayed consequences of exercise-induced muscle tissue injury, cytokine production, and markers of muscle damage has not been investigated. Increased carbohydrate consumption is recommended during recovery to promote glycogen resynthesis; thus, identification of other, perhaps unintended, consequences is important. The purpose of this investigation was to determine whether carbohydrate supplementation during the postexercise recovery period influenced the magnitude of the 
inflammatory and muscle-damage responses to exercise-induced muscle damage. Given the potential for glucose to stimulate TNF- $\alpha$ synthesis and the link between TNF- $\alpha$ and IL-6 produced in response to muscle damage, we hypothesized that carbohydrate supplementation would not attenuate the increase in IL-6 that we measure several hours after our participants completed high-force eccentric exercise. This is contrary to what would be expected with carbohydrate supplementation during endurance exercise. Because this is one of the first studies to investigate the influence of carbohydrate on inflammation and muscle damage during recovery, we focused on the cytokine with the most robust response to exercise and carbohydrate, IL-6; an indication of a systemic acute-phase response, CRP; and a key hormone that is both glucoregulatory and immunomodulatory, cortisol.

\section{Methods}

\section{Participants}

Eight non-weight-trained individuals 19 and 39 years of age completed this investigation. Although there is controversy regarding the existence of sex differences in the response to eccentric exercise, with some studies reporting sex differences and others reporting none (7), each individual acted as his or her own control in this research design; thus both men and women were included in this investigation. Individuals who reported lifting and lowering heavy objects or who recalled experiencing soreness in the arm muscles at any time in the 6 months preceding the investigation were excluded from participation. The aim of these criteria was to eliminate confounding influences of the repeated-bout effect, in which muscle that has been exposed to high-force eccentric exercise will have a blunted muscledamage response to subsequent eccentric-exercise bouts that occur within the next several months (27). Additional exclusion criteria aimed at eliminating potentially confounding conditions included known anemia, musculoskeletal limitations, known inflammatory conditions, diabetes mellitus, heart disease, known kidney problems (excluding kidney stones), smoking, alcohol use greater than 1 drink per day for women and 2 drinks per day for men, binge alcohol drinking (more than 4 drinks on a single occasion), chronic use of anti-inflammatory medications (including over-the-counter nonsteroidal anti-inflammatory drugs), lipid-lowering medications, regular performance of physical activity in which muscle soreness or bruising occurs, and pregnancy. All participants were informed of the risks of participation before giving written informed consent. The study was approved by the Human Subjects Committee at Montana State University.

Data from 8 participants were used in the final statistical analyses. Of the 11 participants who were enrolled in the study, 9 completed and 2 did not complete the study protocol. Large differences between the baseline measures for IL-6 (4-fold difference) and hs-CRP (2-fold difference) were measured in 1 male participant between the 2 conditions. This suggests that inflammation was elevated for reasons unrelated to the investigation before beginning one of the conditions; therefore, this participant was eliminated from the statistical analyses. The remaining participants included in the final statistical analyses were 5 men and 3 women with a mean \pm standard deviation age of $25.0 \pm 7.4 \mathrm{y}$, height of $1.76 \pm 0.1 \mathrm{~m}$, mass of $62.3 \pm$ 
$17.9 \mathrm{~kg}$, and body-mass index of $24.4 \pm 4.0 \mathrm{~kg} / \mathrm{m}^{2}$. Based on data from a previous study, we calculated the sample size needed to detect an effect size of $50-70 \%$ for the magnitude of the IL-6 response as 7-12 participants (6).

\section{Experimental Protocol}

Participants performed a high-force eccentric-exercise bout and then consumed Gatorade (carbohydrate) or an equal volume of placebo during recovery from the exercise. During the carbohydrate condition, participants consumed $0.25 \mathrm{~g}$ of carbohydrate per kilogram of body mass per hour for $12 \mathrm{~h}$ on both the day of and the day after the exercise bout. This dose, when added to the background diet of 50\% carbohydrate, is at the lower end of the range of 7-12 $\mathrm{g}$ of carbohydrate per kilogram of body mass per day recommended to promote optimal glycogen resynthesis during moderate to heavy endurance training (4). During the placebo condition, an equal volume of flavor- and color-matched artificially sweetened placebo was consumed. The carbohydrate and placebo conditions were presented in a balanced order. Starting $1 \mathrm{~d}$ before the exercise and continuing through the first $2 \mathrm{~d}$ after the exercise, participants consumed a controlled diet consisting of 50\% carbohydrate, $30 \%$ protein, and $20 \%$ fat. Caloric intake was determined by averaging the energy intake from a 24-h dietary recall and the Mifflin St.-Joer equation using participant height, weight, age, and self-reported activity level (12). An additional $10 \%$ of calories was added to the obtained average of caloric intake in case a participant desired more food and to address potential underreporting inherent in self-reported dietary assessments. Food for the controlled-diet period was provided to participants by the investigators using the Exchange List for Meal Planning (2). The exercise protocol consisted of baseline assessments at 7:00 AM, followed by a bout of high-force eccentric resistance exercise using the elbow-flexor muscles of the nonpreferred limb (according to self-reported handedness) and follow-up assessments 4, 8, 12, 24,48 , and $120 \mathrm{~h}$ postexercise. Assessments included muscle soreness; midbrachial arm circumference; blood collection for measurement of glucose, IL-6, hs-CRP, cortisol, and creatine-kinase (CK) activity; and maximal force production. The carbohydrate and placebo conditions were separated by 3-6 wk.

Several restrictions were placed on participants to minimize variability in physiological status. Standardized conditions for blood collections in the morning included an overnight fast and minimal physical activity before reporting to the laboratory at 7:00 AM for blood collection and assessments. Strenuous physical exercise, that judged to be near maximal in intensity or longer than $60 \mathrm{~min}$ in duration, was not allowed during the course of the exercise and blood-collection protocols. To minimize the influence of cyclical hormonal variations, all women in the study began each exercise and control protocol within $4 \mathrm{~d}$ of the onset of menstrual bleeding. In addition, to avoid the influence of illness on inflammatory parameters, participants were only tested if they had been free of known infection for at least $1 \mathrm{wk}$.

\section{High-Force Eccentric Exercise}

High-force eccentric exercise was used to induce muscle damage using the method of Clarkson et al. $(8,9)$. The initiation of this damage goes unnoticed, but the ensuing 
inflammatory response leads to delayed-onset muscle soreness beginning 8-24 h after the damage is initiated (21). The protocol for inducing muscle damage in the flexor muscles (primarily $\mathrm{m}$. biceps brachii and $\mathrm{m}$. brachialis) of the nonpreferred arm (arm not used for writing) was performed using a computer-controlled isokinetic dynamometer (Kin Com $125 \mathrm{E}+$, Chattecx Corp., Chattanooga, TN). The dynamometer was adjusted to the body height and limb length of each individual. The arm was supported by a padded bench at approximately 0.79 radians (rad) of shoulder abduction, the axis of rotation of the dynamometer was aligned with the axis of rotation of the elbow, and the forearm was secured to the lever arm of the dynamometer with padded support just proximal to the wrist joint. Three sets of 15 repetitions of eccentric elbow flexion were performed with maximal effort at a rate of 1 repetition per $15 \mathrm{~s}$, with 5 min rest between sets. Repetitions began with the elbow fully flexed and ended with it fully extended. Using maximal effort, participants attempted to keep the elbow in the fully flexed position as the dynamometer pulled the arm to a fully extended position at an angular velocity of $0.79 \mathrm{rad} / \mathrm{s}$, which took approximately $3 \mathrm{~s}$. The dynamometer returned the arm to the fully flexed position and paused for $10 \mathrm{~s}$ before beginning the next repetition. Participants were verbally encouraged to give a maximal effort with each repetition.

\section{Maximal Force Production}

Maximal isometric force production for elbow flexion at an enclosed elbow angle of $1.57 \mathrm{rad}$ was measured before and immediately, $24 \mathrm{~h}, 48 \mathrm{~h}, 96 \mathrm{~h}$, and $120 \mathrm{~h}$ postexercise. To perform the isometric strength measurement, the dynamometer (Kin Com 125 E+, Chattecx Corp., Chattanooga, TN) and subject position were adjusted as for the high-force eccentric exercise, and the lever arm was fixed such that the elbow was positioned with a 1.57-rad enclosed angle. Participants were instructed to pull (flex) for $3 \mathrm{~s}$ using maximal effort. Three maximal efforts were performed with 30 s rest between repetitions. Peak-force measurements from the 3 repetitions were averaged for further analysis. To ensure uniformity of measurements from day to day, all dynamometer position settings for each subject were recorded and reproduced at each testing session.

\section{Muscle Soreness and Swelling}

A subjective assessment of muscle soreness was made by participants using a 100$\mathrm{mm}$ visual analog scale anchored at one end with no soreness and at the other end with very, very sore. Participants were instructed to fully flex and extend the elbow while holding a 1-kg weight and gently squeezing the elbow-flexor muscles and then to place a tick mark on the analog scale that represented the degree of soreness. Furthermore, participants were instructed to think of their ratings in terms of muscle soreness, not of fatigue or relative to other types of pain such as a broken bone. Swelling was assessed by measuring the circumference of the midbrachium (midbiceps) and at a point halfway between the midbiceps and the axis of rotation of the elbow using a spring-loaded anthropometric tape measure. The peak of the biceps brachii muscle was palpated in a flexed position, and 3 ink dots were placed on the arm in a plane perpendicular to the axis of the limb. These marks were maintained throughout the protocol and used to guide consistent tape-measure placement. 


\section{Blood Collection and Analysis}

Participants were seated for 10-15 min before blood was collected from an antecubital vein into evacuated tubes using a standard venipuncture technique. Blood was collected in a vacuum tube without additive for analysis of hs-CRP, cortisol, and $\mathrm{CK}$ and containing EDTA for IL-6. After clotting, serum was separated from cells using a refrigerated 21000R Marathon centrifuge (Fisher Scientific, Pittsburgh, PA). All samples were stored at $-80^{\circ} \mathrm{C}$ until analysis.

Serum hs-CRP (MP Biomedicals, Irvine, CA), plasma IL-6 (R\&D Systems, Minneapolis, MN), and serum cortisol (Diagnostic Systems Laboratories, Inc., Webster, TX) concentrations were measured using commercially available ELISA kits according to the manufacturers' instructions. Absorbance of 96-well assay plates was read at the primary wavelength and corrected for secondary-wavelength interference using a $\mu$ Quant Universal microplate spectrophotometer (Bio-Tek Instruments, Winooski, VT). All samples were run in duplicate. Average intraassay coefficients of variation for hs-CRP, IL-6, and cortisol were $12.0 \%, 9.6 \%$, and $3.0 \%$, respectively. All samples for a given participant were run on the same assay plate.

Serum CK activity was measured using an ultraviolet kinetic assay (Thermo Electron Corp., Melbourne, Australia). Serum glucose concentration was assayed using a glucose hexokinase reagent set (Pointe Scientific, Inc., Canton, MI). These assays were modified for microplate analysis and read using the $\mu$ Quant Universal microplate spectrophotometer. Samples were run in duplicate. The intra-assay coefficients of variation were $2.1 \%$ and $3.1 \%$ for $\mathrm{CK}$ and glucose, respectively. All samples for a given participant were analyzed in the same run of the assay.

\section{Statistical Analysis}

Data were analyzed using the Statistical Program for Social Sciences (SPSS) for Windows (version 13.0, SPSS Inc., Chicago, IL). Baseline measures were compared between groups using an independent-sample $t$ test for continuous variables. A general linear-model 2-way repeated-measures analysis of variance (ANOVA) was used to compare conditions (carbohydrate and placebo) over time. The Greenhouse-Geisser adjustment to degrees of freedom was used when the assumption of sphericity was violated. All variables were normally distributed according to the Kolmogorov-Smirnov test; therefore, no transformations were needed in the analysis. Statistica version 6.0 software (Statsoft, Inc., Tulsa, OK) was used to perform Tukey's honestly significant difference post hoc analysis to determine the location of differences when significant main effects or interactions were detected. Statistical significance was set a priori at the alpha $=0.05$ level.

\section{Results}

\section{Background Diet and Blood Glucose Across Conditions}

We controlled the background diet between conditions, but not the timing of food consumption. Participants were given food packets with the appropriate macronutrient proportions and asked to record how much of the food they consumed. As a result, participants were consistent in their macronutrient intake across conditions and very close to the background-diet target of $50 \%$ carbohydrate, $20 \%$ protein, 
and 30\% fat (Table 1). In 1 instance, a participant (subject 7) appeared to compensate for the increased carbohydrate intake during that condition by decreasing her intake of carbohydrate in the background diet. The timing of food ingestion for the background diet was not controlled. For reasons that might include variability in timing of background food consumption, serum glucose concentrations were not higher in the carbohydrate than in the placebo condition (Figure 1).

\section{Table 1 Average Energy and Carbohydrate (CHO) Intake per Day for the Day of and the Day After the Eccentric-Exercise Bout in the Placebo (P) and Carbohydrate (C) Conditions}

\begin{tabular}{|c|c|c|c|c|c|c|c|}
\hline Subject & $\begin{array}{l}\text { Mass } \\
(\mathrm{kg})\end{array}$ & $\begin{array}{c}\text { Total } \\
\text { energy P } \\
\text { (kcal) }\end{array}$ & $\begin{array}{l}\text { CHO P } \\
\text { (kcal) }\end{array}$ & $\begin{array}{c}\text { CHO P } \\
\left(\mathrm{g} \cdot \mathrm{kg}^{-1} \cdot \mathrm{d}^{-1}\right)\end{array}$ & $\begin{array}{c}\text { Total } \\
\text { energy } \\
\text { C (kcal) }\end{array}$ & $\begin{array}{l}\text { CHO C } \\
\text { (kcal) }\end{array}$ & $\begin{array}{c}\text { CHO C } \\
\left(\mathrm{g} \cdot \mathrm{kg}^{-1} \cdot \mathrm{d}^{-1}\right)\end{array}$ \\
\hline 1 & 78 & 3717 & 1892 & 6.1 & 4531 & 2764 & 8.9 \\
\hline 2 & 76 & 2772 & 1549 & 5.1 & 3952 & 2606 & 8.6 \\
\hline 3 & 74 & 2776 & 1544 & 5.2 & 3511 & 2315 & 7.8 \\
\hline 4 & 89 & 1654 & 975 & 2.7 & 2692 & 2024 & 5.7 \\
\hline 5 & 54 & 2131 & 1178 & 5.5 & 2307 & 1563 & 7.2 \\
\hline 6 & 93 & 1440 & 660 & 1.8 & 2844 & 1974 & 5.3 \\
\hline 7 & 74 & 2047 & 1016 & 3.4 & 2106 & 1429 & 4.8 \\
\hline 8 & 70 & 1905 & 999 & 3.6 & 2742 & 1836 & 6.6 \\
\hline Mean & 69.8 & 2305 & 1226 & 4.6 & 3085 & 2063 & 6.9 \\
\hline $\begin{array}{l}\text { Standard } \\
\text { deviation }\end{array}$ & 16.4 & 743 & 402 & 1.5 & 838 & 472 & 1.5 \\
\hline
\end{tabular}

\section{$\cdots \diamond--$ Placebo $\rightarrow-$ Carbohydrate}

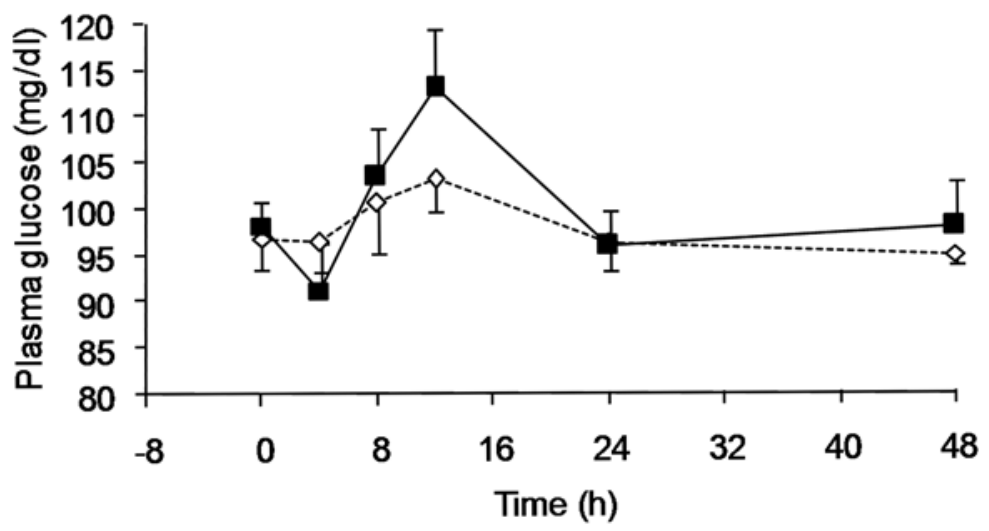

Figure 1 - Serum glucose concentrations between the placebo and carbohydrate conditions. Values are mean \pm standard error of the mean. 


\section{IL-6, CRP, and Cortisol}

Preexercise IL-6 concentrations did not differ between the placebo $(0.81 \pm 0.49$ $\mathrm{pg} / \mathrm{mL})$ and carbohydrate conditions $(0.58 \pm 0.41 \mathrm{pg} / \mathrm{mL})$. Plasma IL-6 concentrations increased, $F(5)=5.27, P<0.05,8 \mathrm{~h}$ after eccentric exercise. There was no difference between the 2 conditions (Figure 2). Preexercise CRP concentrations did not differ between the placebo $(0.90 \pm 1.02 \mathrm{mg} / \mathrm{L})$ and carbohydrate conditions $(0.72 \pm 0.65 \mathrm{mg} / \mathrm{L}) . \mathrm{CRP}$ did not change over time, and no differences were detected between placebo and carbohydrate conditions (Figure 3). The characteristic diurnal

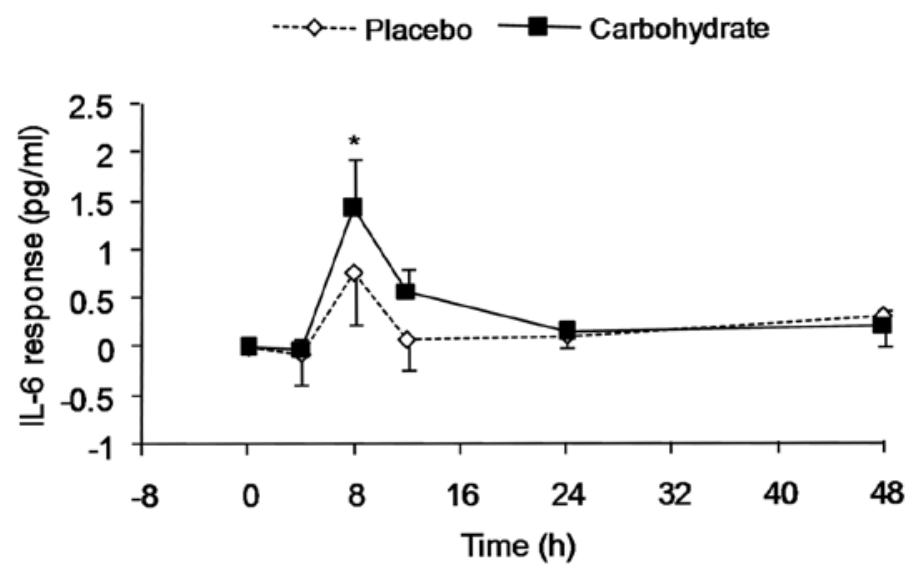

Figure 2 - IL-6 responses (postexercise concentration - preexercise concentration) after eccentric exercise in the placebo and carbohydrate conditions. Values are mean \pm standard error of the mean. $* P<0.05$ compared with preexercise.

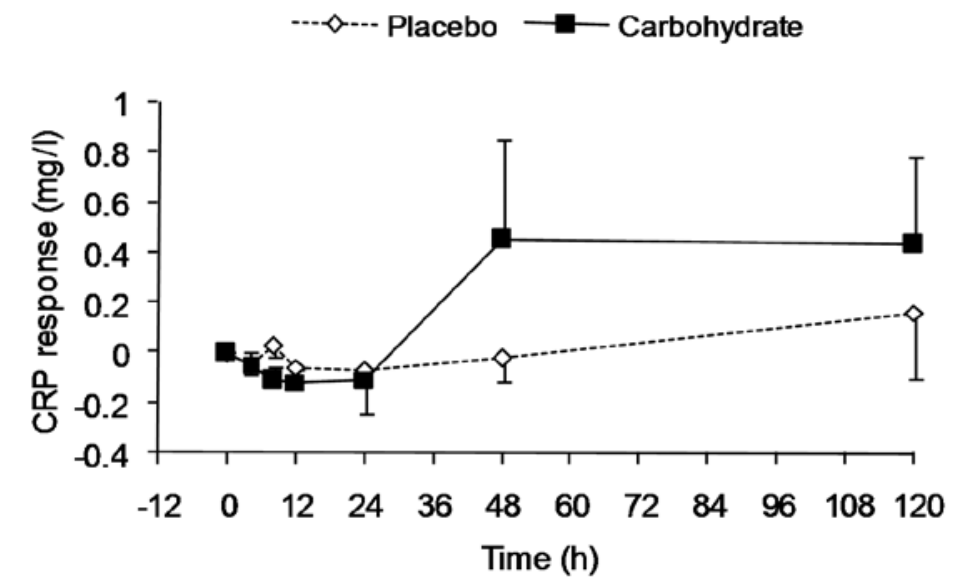

Figure 3 - hs-CRP responses (postexercise concentration - preexercise concentration) after eccentric exercise in the placebo and carbohydrate conditions. Values are mean \pm standard error of the mean. 
---- Placebo - Carbohydrate

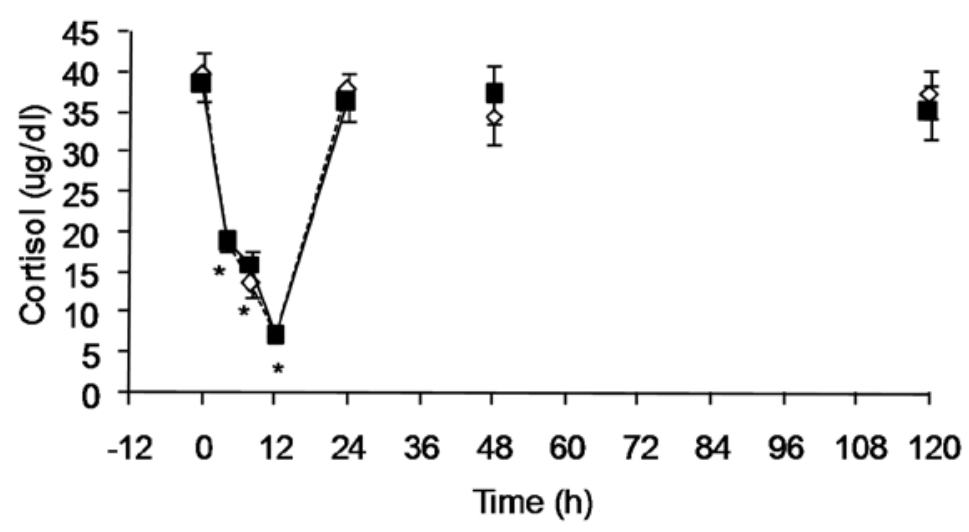

Figure 4 - Cortisol concentrations after eccentric exercise in the placebo and carbohydrate conditions. Values are mean \pm standard error of the mean. $* P<0.05$ compared with preexercise.

variation in serum cortisol concentrations occurred, in which large decreases, $F(6)$ $=63.16, P<0.001$, were measured 4,8 , and $12 \mathrm{~h}$ postexercise (Figure 4 ). These measures were made at 12:00, 4:00, and 8:00 PM, respectively. All other measurements were made at 7:00 AM, the characteristic time for the highest serum cortisol concentrations. No differences between carbohydrate and placebo were found.

\section{Maximal Isometric Force, Swelling, Muscle Soreness, and CK Enzyme Activity}

Subjective ratings of muscle soreness increased, $F(6)=15.60, P<0.001,8-48$ $\mathrm{h}$ postexercise, but there were no differences between placebo and carbohydrate conditions (Figure 5). There was a change, $F(6)=3.54, P<0.001$, in arm circumference at the midbiceps level over time; however, Tukey post hoc analysis did not identify significant differences between means (Figure 6). Maximal isometric force decreased, $F(4)=9.98, P<0.05$, immediately postexercise to $64.5 \%$ and $67.0 \%$ of preexercise values for the placebo and carbohydrate conditions, respectively, but recovered by $24 \mathrm{~h}$ postexercise. No differences in force loss or recovery were measured between conditions, and the correlation in the proportion of strength lost between conditions was $r=0.77$. Serum CK-activity responses varied from subject to subject, and only 2 of the participants had increases that would be considered large, over 1000 IU/L. Placebo and carbohydrate responses were within $15 \%$ of each other for the 2 high-CK respondents.

\section{Discussion}

The purpose of this investigation was to determine whether carbohydrate supplementation during the post-eccentric-exercise recovery period influenced the magnitude of the IL-6, CRP, cortisol, and muscle-damage-marker responses. The key 


\section{$\cdots \diamond$ Placebo $\rightarrow-$ Carbohydrate}

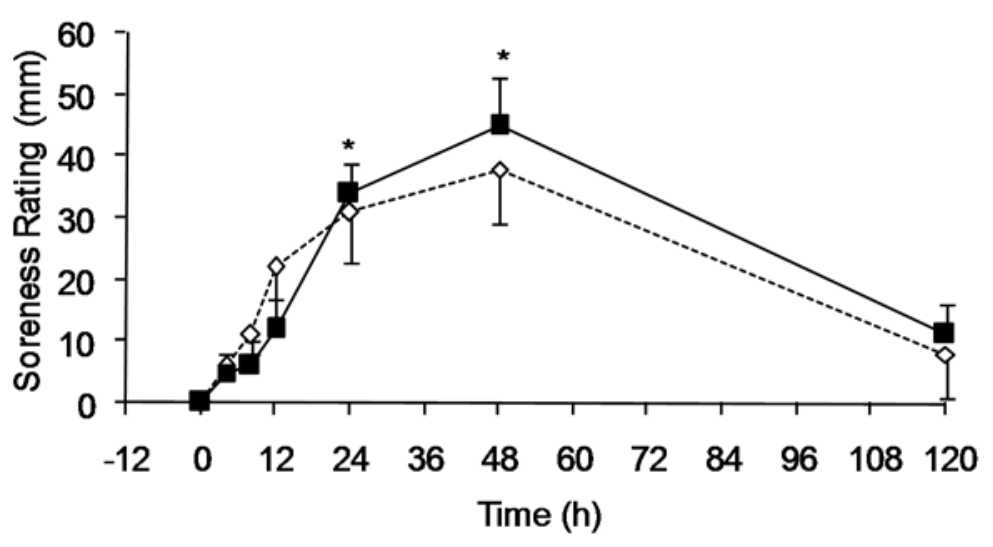

Figure 5 - Ratings of muscle soreness on a 100-mm visual analog scale anchored with no soreness at one end and very, very sore at the other end. Values are mean \pm standard error of the mean. ${ }^{*} P<0.05$ compared with preexercise.

\section{$\cdots$ Placebo $\rightarrow-$ Carbohydrate}

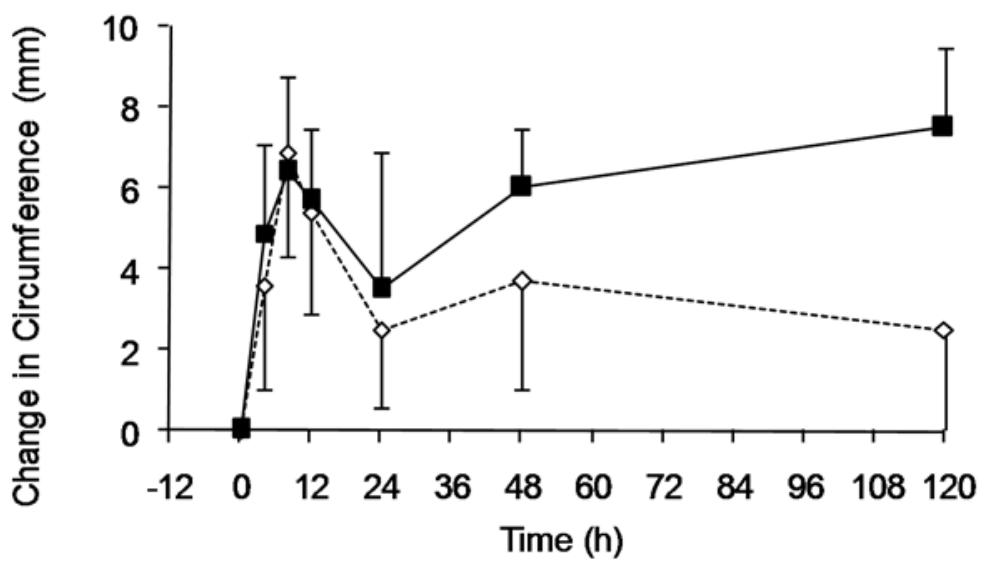

Figure 6 - Swelling at the midbiceps level after eccentric exercise for the placebo and carbohydrate conditions. The main effect for time was significant; however, Tukey post hoc analysis did not identify significant differences relative to preexercise. Values are mean \pm standard error of the mean.

finding of this investigation is that carbohydrate supplementation during recovery from muscle-soreness-inducing exercise did not attenuate the IL-6 response that occurs several hours postexercise. Although carbohydrate supplementation during prolonged exercise suppresses the IL-6 response during and immediately after the 
exercise $(23,25,26)$, the current study is the first to evaluate the influence of carbohydrate during recovery from high-force eccentric exercise when IL-6 production temporally linked to the inflammatory process is likely to be taking place.

The 2 high-force eccentric-exercise bouts elicited similar decreases in maximal isometric force immediately postexercise, suggesting that the exercise stress between conditions was comparable. The magnitude of the muscle-damage response was modest and likely to be similar to that following an exercise-training bout and smaller than that after a strenuous competition or unaccustomed exercise bout. More specifically, the exercise bout was one in which delayed-onset muscle soreness was at a moderate level for a few days postexercise, but the loss of muscle function was not prolonged. Although other studies using eccentric-exercise models have reported prolonged strength losses $(7,27)$, the use of the isokinetic exercise dynamometer in the current study and the possibility of previous exposure to high eccentric forces in the elbow-flexor muscles that participants were not aware of or did not report might account for the relatively small magnitude of this response relative to those in other studies.

There are 2 independent pathways in which most exercise-induced IL- 6 production occurs, one that leads to production by working muscle cells and the other to production in response to tissue injury. IL- 6 from working muscle tissue occurs independent of tissue damage, does not include production of the inflammatory cytokine tumor-necrosis factor- $\alpha$ (TNF- $\alpha)$, is associated with substrate metabolism and glucose regulation, reaches peak plasma concentrations during or immediately after the completion of the exercise, and declines rapidly to resting levels early in recovery $(10,16,30,33,34)$. IL-6 produced in response to muscle damage is smaller in magnitude relative to IL-6 from working muscle, reaches peak plasma concentrations hours after completion of exercise, and might remain elevated for several days after the initiation of inflammation $(17,30)$. Initiation of muscle-tissue injury promotes infiltration by inflammatory cells that in conjunction with local muscle, endothelial, and satellite cells produce an array of cytokines to regulate the inflammatory process, including TNF- $\alpha$, IL-1 $\beta$, and IL-6 $(5,30,32,35)$. The approximately 2 -fold increase in IL-6 eight hours postexercise in the current study is consistent with production of IL-6 in response to muscle injury.

In contrast to our finding that carbohydrate supplementation during the recovery period did not attenuate the delayed increase in plasma IL-6, several other studies have clearly demonstrated that carbohydrate supplementation during endurance exercise attenuates increases in several mediators of stress and inflammation, including cortisol and IL-6 $(23,26)$. Carbohydrate supplementation during exercise is typically at a dose of approximately $0.96 \mathrm{~g}$ of carbohydrate per kilogram of body weight per hour during the course of the exercise bout, but lower ad libitum intakes also have been shown to attenuate the IL-6 response (22). This supplementation regimen dramatically attenuated the IL- 6 response and the responses of antiinflammatory mediators induced by IL-6, for example, IL-1 receptor antagonist (IL-1ra) and IL-10 (23, 26). Major differences to be considered between these studies and the current investigation include the type of exercise (endurance vs. resistance), the dose of carbohydrate $\left(0.96 \mathrm{vs} .0 .25 \mathrm{~g} \cdot \mathrm{kg}^{-1} \cdot \mathrm{h}^{-1}\right)$, and the timing of carbohydrate supplementation (before and during vs. after).

Unlike as in endurance exercise, carbohydrate supplementation or status does not influence cytokine responses to resistance exercise. Nieman et al. (24) clearly 
demonstrated that muscle cytokine gene expression and plasma cytokine levels during and $1 \mathrm{~h}$ after a 2-h intensive resistance-exercise bout were not influenced by carbohydrate supplementation during the exercise bout. The authors suggested that this is consistent with other research in which the exercise is intermittent. That study did not evaluate the delayed increase in IL-6, and the supplementation only before and during the course of the exercise bout. The findings of the current study are consistent with those of Nieman et al., however, in that we did not measure differences in IL- 6 responses between carbohydrate and placebo conditions in response to resistance exercise.

Relative to studies in which attenuation of inflammatory cytokine production during exercise was measured, our study used a lower rate of carbohydrate consumption, occurred over a longer period of time, and occurred during the recovery period. Participants consumed $0.25 \mathrm{~g}$ of carbohydrate per kilogram of body mass per hour from $0-12$ and $24-36 \mathrm{~h}$ postexercise. This supplementation occurred in addition to a background diet in which total caloric and macronutrient composition were equal for each participant across conditions. Thus, the overall dose of carbohydrate above the background diet was $6 \mathrm{~g}$ of carbohydrate per kilogram of body mass (equivalent to $373.8 \pm 107.4 \mathrm{~g}$ total, or $1495 \pm 430 \mathrm{kcal}$ total) over the course of $2 \mathrm{~d}$. The rate of carbohydrate administration was relatively slow and did not raise blood glucose levels significantly. Allowing participants to consume the background diet whenever they chose to also might have contributed to the lack of difference in blood glucose concentrations between carbohydrate and placebo conditions.

CRP is an inflammatory protein made by the liver in response to increases in IL-6 and other inflammatory mediators $(3,15)$. Rises in CRP indicate that the IL-6 produced at the tissue level is triggering an acute-phase, systemic inflammatory response (3). Increases in CRP approximately 24-72 h postexercise have been measured after exercise in which elevations in serum $\mathrm{CK}$ activity also were present $(13,31)$. In the current investigation, a consistent increase in serum CK activity was not measured, and the IL-6 increase was not sufficient to induce a systemic inflammatory response; thus CRP did not increase in either the carbohydrate or the placebo condition after the exercise.

Cortisol is a stress hormone that has a glucoregulatory function and interacts with IL-6 $(20,28)$. An inverse relationship between blood glucose and cortisol concentrations and lower cortisol responses during endurance exercise with carbohydrate supplementation has been established (23). We measured this hormone so that we could determine whether the spike in IL-6 or the carbohydrate supplementation influenced cortisol levels. There were no differences in cortisol concentrations between carbohydrate and placebo conditions. Thus, the normal diurnal pattern of cortisol fluctuation was not influenced by the addition of a carbohydrate supplement.

We conclude that carbohydrate supplementation during recovery from sorenessinducing resistance exercise does not influence the delayed IL-6 response temporally linked to the inflammatory response to tissue injury. This conclusion should be regarded with caution until confirmed with additional data from studies employing larger sample sizes. Similarly, markers of muscle damage were not influenced by the ingestion of carbohydrate. The level of muscle injury was low and the rate of carbohydrate supplementation was low, so further research is needed to determine 
whether this conclusion is consistent when greater amounts of inflammation and faster rates of glucose supplementation occur.

\section{Acknowledgments}

This study was supported by a grant from the Gatorade Sports Science Institute (GSSI).

\section{References}

1. Aljada, A., J. Friedman, H. Ghanim, et al. Glucose ingestion induces an increase in intranuclear nuclear factor kappaB, a fall in cellular inhibitor kappaB, and an increase in tumor necrosis factor alpha messenger RNA by mononuclear cells in healthy human subjects. Metabolism. 55:1177-1185, 2006.

2. American Diabetes Association and American Dietetic Association. Exchange List for Meal Plan. 2nd ed. Chicago: American Diabetes Association and American Dietetic Association, 2003.

3. Baumann, H., and J. Gauldie. The acute phase response. Immunol. Today. 15:74-80, 1994.

4. Burke, L.M., B. Kiens, and J.L. Ivy. Carbohydrates and fat for training and recovery. J. Sports Sci. 22:15-30, 2004.

5. Cannon, J.G., and B.A. St. Pierre. Cytokines in exertion-induced skeletal muscle injury. Mol. Cell. Biochem. 179:159-167, 1998.

6. Chow, S-C., J. Shao, and H. Wang. Sample Size Calculations in Clinical Research. New York: Taylor \& Francis, 2003, pp. 64-65.

7. Clarkson, P.M., and M.J. Hubal. Exercise-induced muscle damage in humans. Am. J. Phys. Med. Rehabil. 81:S52-S69, 2002.

8. Clarkson, P.M., K. Nosaka, and B. Braun. Muscle function after exercise-induced muscle damage and rapid adaptation. Med. Sci. Sports Exerc. 24:512-520, 1992.

9. Clarkson, P.M., and I. Tremblay. Exercise-induced muscle damage, repair, and adaptation in humans. J. Appl. Physiol. 65:1-6, 1988.

10. Febbraio, M.A., and B.K. Pedersen. Muscle-derived interleukin-6: mechanisms for activation and possible biological roles. FASEB J. 16:1335-1347, 2002.

11. Fielding, R.A., T.J. Manfredi, W. Ding, M.A. Fiatarone, W.J. Evans, and J.G. Cannon. Acute phase response in exercise, III: neutrophil and IL-1 beta accumulation in skeletal muscle. Am. J. Physiol. 265:R166-R172, 1993.

12. Frankenfield, D.C., W.A. Rowe, J.S. Smith, and R.N. Cooney. Validation of several established equations for resting metabolic rate in obese and nonobese people. J. Am. Diet. Assoc. 103:1152-1159, 2003.

13. Gleeson, M., J. Almey, S. Brooks, R. Cave, A. Lewis, and H. Griffiths. Haematological and acute-phase responses associated with delayed-onset muscle soreness in humans. Eur. J. Appl. Physiol. Occup. Physiol. 71:137-142, 1995.

14. Gonzalez, F., J. Minium, N.S. Rote, and J.P. Kirwan. Altered tumor necrosis factor alpha release from mononuclear cells of obese reproductive-age women during hyperglycemia. Metabolism. 55:271-276, 2006.

15. Guillen, I., M. Blanes, M.J. Gomez-Lechon, and J.V. Castell. Cytokine signaling during myocardial infarction: sequential appearance of IL-1 beta and IL-6. Am. J. Physiol. 269:R229-R235, 1995.

16. Helge, J.W., B. Stallknecht, B.K. Pedersen, H. Galbo, B. Kiens, and E.A. Richter. The effect of graded exercise on IL-6 release and glucose uptake in human skeletal muscle. J. Physiol. 546:299-305, 2003.

17. Hellsten, Y., U. Frandsen, N. Orthenblad, B. Sjodin, and E.A. Richter. Xanthine oxidase in human skeletal muscle following eccentric exercise: a role in inflammation. $J$. Physiol. 498(Pt. 1):239-248, 1997. 
18. Kasim-Karakas, S.E., A. Tsodikov, U. Singh, and I. Jialal. Responses of inflammatory markers to a low-fat, high-carbohydrate diet: effects of energy intake. Am. J. Clin. Nutr. 83:774-779, 2006.

19. MacIntyre, D.L., S. Sorichter, J. Mair, A. Berg, and D.C. McKenzie. Markers of inflammation and myofibrillar proteins following eccentric exercise in humans. Eur. J. Appl. Physiol. 84:180-186, 2001.

20. Miles, M.P. Neuroendocrine modulation of the immune system with exercise and muscle damage. In: The Endocrine System in Sports and Exercise, W.J. Kraemer and A.D. Rogol (Eds.). Oxford, UK: Blackwell, 2005, pp. 345-367.

21. Miles, M.P., and P.M. Clarkson. Exercise-induced muscle pain, soreness, and cramps. J. Sports Med. Phys. Fitness. 34:203-216, 1994.

22. Miles, M.P., E.E. Walker, S.B. Conant, S.P. Hogan, and J.R. Kidd. Carbohydrate influences plasma interleukin-6 but not C-reactive protein or creatine kinase following a 32-km mountain trail race. Int. J. Sport Nutr. Exerc. Metab. 17:36-46, 2006.

23. Nehlsen-Cannarella, S.L., O.R. Fagoaga, D.C. Nieman, et al. Carbohydrate and the cytokine response to 2.5 h of running. J. Appl. Physiol. 82:1662-1667, 1997.

24. Nieman, D.C., J.M. Davis, V.A. Brown, et al. Influence of carbohydrate ingestion on immune changes after $2 \mathrm{~h}$ of intensive resistance training. J. Appl. Physiol. 96:12921298, 2004.

25. Nieman, D.C., J.M. Davis, D.A. Henson, et al. Muscle cytokine mRNA changes after $2.5 \mathrm{~h}$ of cycling: influence of carbohydrate. Med. Sci. Sports Exerc. 37:1283-1290, 2005.

26. Nieman, D.C., J.M. Davis, D.A. Henson, et al. Carbohydrate ingestion influences skeletal muscle cytokine mRNA and plasma cytokine levels after a 3-h run. J. Appl. Physiol. 94:1917-1925, 2003.

27. Nosaka, K., P.M. Clarkson, M.E. McGuiggin, and J.M. Byrne. Time course of muscle adaptation after high force eccentric exercise. Eur. J. Appl. Physiol. Occup. Physiol. 63:70-76, 1991.

28. Papanicolaou, D.A., J.S. Petrides, C. Tsigos, et al. Exercise stimulates interleukin-6 secretion: inhibition by glucocorticoids and correlation with catecholamines. Am. J. Physiol. 271:E601-E605, 1996.

29. Paulsen, G., H.B. Benestad, I. Strom-Gundersen, L. Morkrid, K.T. Lappegard, and T. Raastad. Delayed leukocytosis and cytokine response to high-force eccentric exercise. Med. Sci. Sports Exerc. 37:1877-1883, 2005.

30. Pedersen, B.K., A. Steensberg, and P. Schjerling. Muscle-derived interleukin-6: possible biological effects. J. Physiol. 536:329-337, 2001.

31. Phillips, T., A.C. Childs, D.M. Dreon, S. Phinney, and C. Leeuwenburgh. A dietary supplement attenuates IL-6 and CRP after eccentric exercise in untrained males. Med. Sci. Sports Exerc. 35:2032-2037, 2003.

32. Smith, L.L., and M.P. Miles. Exercise-induced muscle injury and inflammation. In: Exercise and Sport Science, W.E. Garrett and D.T. Kirkendall (Eds.). Philadelphia: Lippincott Williams \& Wilkins, 2000, pp. 401-411.

33. Steensberg, A., C. Keller, R.L. Starkie, T. Osada, M.A. Febbraio, and B.K. Pedersen. IL-6 and TNF-alpha expression in, and release from, contracting human skeletal muscle. Am. J. Physiol. Endocrinol. Metab. 283:E1272-E1278, 2002.

34. Steensberg, A., G. van Hall, T. Osada, M. Sacchetti, B. Saltin, and B. Klarlund Pedersen. Production of interleukin-6 in contracting human skeletal muscles can account for the exercise-induced increase in plasma interleukin-6. J. Physiol. 529(Pt. 1):237-242, 2000.

35. Tomiya, A., T. Aizawa, R. Nagatomi, H. Sensui, and S. Kokubun. Myofibers express IL-6 after eccentric exercise. Am. J. Sports Med. 32:503-508, 2004. 